\title{
Novel Pauli-paramagnetic quantum phase in a Mott insulator
}

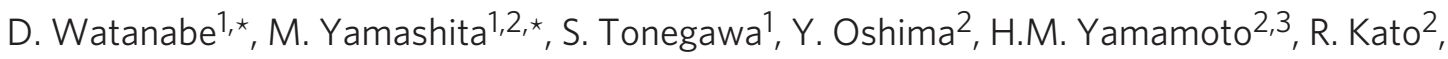

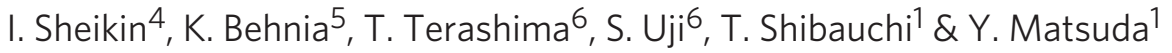

In Mott insulators, the strong electron-electron Coulomb repulsion localizes electrons. In dimensions greater than one, their spins are usually ordered antiferromagnetically at low temperatures. Geometrical frustrations can destroy this long-range order, leading to exotic quantum spin liquid states. However, their magnetic ground states have been a longstanding mystery. Here we show that a quantum spin liquid state in the organic Mott insulator EtMe $3 \mathrm{Sb}\left[\mathrm{Pd}(\mathrm{dmit})_{2}\right]_{2}$ (where $\mathrm{Et}$ is $\mathrm{C}_{2} \mathrm{H}_{5}-$, Me is $\mathrm{CH}_{3}-$, and dmit is 1,3-dithiole-2-thione-4, 5-dithiolate) with two-dimensional triangular lattice has Pauli-paramagnetic-like low-energy excitations, which are a hallmark of itinerant fermions. Our torque magnetometry down to low temperatures $(30 \mathrm{mK})$ up to high fields $(32 \mathrm{~T})$ reveals distinct residual paramagnetic susceptibility comparable to that in a half-filled two-dimensional metal, demonstrating the magnetically gapless nature of the ground state. Moreover, our results are robust against deuteration, pointing toward the emergence of an extended 'quantum critical phase', in which low-energy spin excitations behave as in paramagnetic metals with Fermi surface, despite the frozen charge degree of freedom.

\footnotetext{
${ }^{1}$ Department of Physics, Kyoto University, Kyoto 606-8502, Japan. ${ }^{2}$ RIKEN, Wako, Saitama 351-0198, Japan. ${ }^{3}$ Institute for Molecular Science, Myodaiji, Okazaki 444-8585, Japan. ${ }^{4}$ Grenoble High Magnetic Field Laboratory, CNRS, 38042 Grenoble Cedex 9, France. ${ }^{5}$ LPEM (CNRS-UPMC), Ecole Supérieure de Physique et de Chimie Industrielles, 75005 Paris, France. ${ }^{6}$ National Institute for Materials Science, Ibaraki 305-0003, Japan. ${ }^{\star}$ These authors contributed equally to this work. Correspondence and requests for materials should be addressed to T.S. (email: shibauchi@scphys.kyoto-u.ac.jp) or to Y.M. (email: matsuda@scphys.kyoto-u.ac.jp).
} 
A t sufficiently low temperatures, condensed matter systems generally tend to order. Quantum spin liquids (QSLs) ${ }^{1}$ are a prominent exception, in which no local order parameter is formed while the entropy vanishes at zero temperature. In two- or three-dimensional (2D or $3 \mathrm{D})$ systems, it is widely believed ${ }^{1}$ that QSL ground states may emerge when interactions among the magnetic degrees of freedom are incompatible with the underlying crystal geometry. Typical 2D examples of such geometrically frustrated systems can be found in triangular and kagome lattices. Largely triggered by the proposal of the resonating-valence-bond theory on spin-1/2 degrees of freedom residing on a $2 \mathrm{D}$ triangular lattice ${ }^{2}$ and its possible application to high-transition-temperature superconductivity $^{3}$, realizing/detecting QSLs has been a long-sought goal. Until now, quite a number of QSLs with various types of ground states have been proposed ${ }^{2,4-19}$, but the lack of real materials had prevented us from understanding the nature of QSLs.

The two recently discovered organic Mott insulators, $\kappa$-(BEDTTTF $)_{2} \mathrm{Cu}_{2}(\mathrm{CN})_{3}$ (refs 20-25) and $\mathrm{EtMe}_{3} \mathrm{Sb}\left[\mathrm{Pd}(\mathrm{dmit})_{2}\right]_{2}$ (refs 26-29) (see Fig. 1a), are very likely to be the first promising candidates to host a QSL in real bulk materials ${ }^{30}$. In these systems, $(\text { BEDT-TTF) })^{1 / 2+}$ cations or $\mathrm{Pd}(\mathrm{dmit})_{2}^{1 / 2-}$ anions are strongly dimerized and spin-1/2 units of (BEDT-TTF $)_{2}$ and $\left[\mathrm{Pd}(\mathrm{dmit})_{2}\right]_{2}$ form $2 \mathrm{D}$ triangular structure. In both systems, nuclear magnetic resonance (NMR) 20,26 and muon spin rotation ${ }^{24,25}$ measurements exhibit no sign of long-range magnetic ordering down to a very low temperature whose energy scale corresponds to $J / 10,000$ ( $J / k_{\mathrm{B}} \sim 250 \mathrm{~K}$ for both compounds), where $J$ is the nearest-neighbour exchange coupling energy. These findings aroused great interest because even in a quantum spin-1/2 triangular lattice antiferromagnet, the frustration brought on by the nearestneighbour Heisenberg interaction is known to be insufficient to destroy the long-range ordered ground state ${ }^{31}$. Several classes of QSL states have been put forth to explain these exotic spin states, yet their ground state remains puzzling. Between the two compounds, $\mathrm{EtMe}_{3} \mathrm{Sb}\left[\mathrm{Pd}(\mathrm{dmit})_{2}\right]_{2}$ appears to be more ideal to single out genuine features of the QSL, because more homogeneous QSL state can be attained at low temperatures ${ }^{28}$. In contrast, $\kappa$-(BEDT$\mathrm{TTF})_{2} \mathrm{Cu}_{2}(\mathrm{CN})_{3}$ has been reported to have a structural transition at $6 \mathrm{~K}$ (ref. 23) and an inhomogeneous, phase-separated spin state ${ }^{20,24}$.

What is remarkable in the QSL state of $\mathrm{EtMe}_{3} \mathrm{Sb}\left[\mathrm{Pd}(\mathrm{dmit})_{2}\right]_{2}$ is the presence of the gapless excitations, which are highly mobile with long mean-free path, as reported by thermal conductivity ${ }^{27}$ and heat capacity measurements ${ }^{29}$. However, there are two fundamental questions that still remain open. The first one is the magnetic nature of the gapless excitations, which cannot be obtained from the above measurements. Whether the excitations are magnetic or nonmagnetic bears direct implications on the spin-spin correlation function. The second question concerns the phase diagram; how the QSL varies when tuned by the non-thermal parameter, such as frustration, is the key to understanding the ground state. The uniform susceptibility in the low-temperature limit provides pivotal information on the magnetic character of the ground state. However, conventional measurements using SQUID magnetometer are susceptible to paramagnetic contributions due to impurities. Indeed, for $\mathrm{EtMe}_{3} \mathrm{Sb}\left[\mathrm{Pd}(\mathrm{dmit})_{2}\right]_{2}$, the temperature region of importance is below $\sim 5 \mathrm{~K}$, but SQUID signal is dominated by the paramagnetic contributions residing on the non-magnetic cation $\mathrm{EtMe}_{3} \mathrm{Sb}$ layers ${ }^{27}$.

To resolve the intrinsic magnetic susceptibility of the QSL in single-crystalline samples, we performed the magnetic torque $\tau$ measurements by using a micro-cantilever method ${ }^{32,33}$ (Fig. 1b). The torque is given by the cross product of the applied field $H$ and the magnetization $M\left(M_{i}=\Sigma_{j} \chi_{i j} H_{j}\right.$, where $\chi_{i j}$ is the spin susceptibility tensor diagonalised along the magnetic principal axes, see Fig. $1 \mathrm{c}, \mathrm{d})$ as $\tau=\mu_{0} V M \times H$, where $\mu_{0}$ is the permeability of vacuum and $V$ is the sample volume. The most advantage of this method is that the large Curie contribution from impurity spins is cancelled
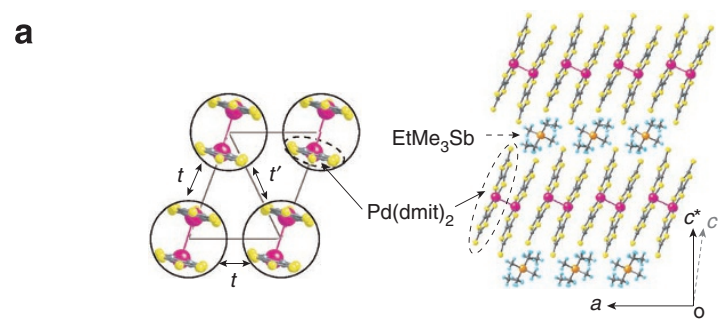

b

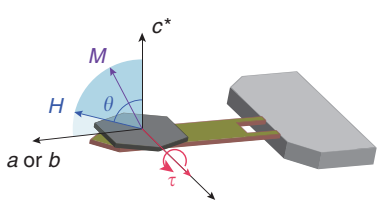

C

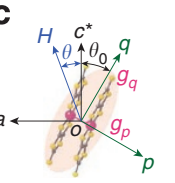

d
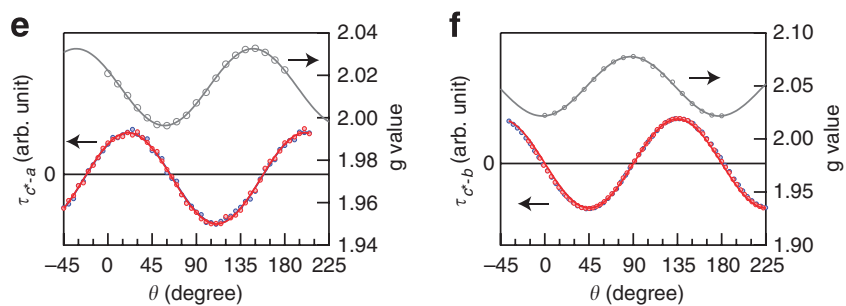

Figure 1 | Magnetic torque measurements in the QSL state of the organic Mott insulator with 2D triangular lattices. (a) The crystal structure of $\mathrm{EtMe}_{3} \mathrm{Sb}\left[\mathrm{Pd}(\mathrm{dmit})_{2}\right]_{2}$ that viewed from the $b$ axis (right). The crystal consists of $2 \mathrm{D}$ layers of $\mathrm{Pd}(\mathrm{dmit})_{2}$ molecules separated by the nonmagnetic cation $\mathrm{EtMe}_{3} \mathrm{Sb}$. The $\mathrm{c}^{\star}$ axis is defined as a perpendicular axis to the $a b$ plane. In the $\mathrm{Pd}(\mathrm{dmit})_{2}$ layer, the face-to-face pair of $\mathrm{Pd}(\mathrm{dmit})_{2} 1 / 2$ anions are strongly dimerised (encircled) and spin-1/2 (one electron) units of encircled $\left[\mathrm{Pd}(\mathrm{dmit})_{2}\right]_{2}$ form the triangular lattice. The ratio of the nearest-neighbour inter-dimer transfer integrals is estimated to be $t^{\prime} / t=0.92$ from the tight-binding calculations based on the crystallographic data at room temperatures, indicating a nearly ideal triangular structure. (b) Schematic drawing of torque measurements using a cantilever. By rotating magnetic field from the $c^{\star}$ axis to $a$ or $b$ axis, the magnetic torque component perpendicular to the rotating field plane is measured as a function of the polar angle $\theta$. (c,d) The gyromagnetic ratio ( $g$-factor) of $\left[\operatorname{Pd}(\mathrm{dmit})_{2}\right]_{2}$ with spin-1/2. The principal axes of $g$-tensor denoted by $p, q$ and $r(\| \mathrm{b})$ do not coincide with crystallographic axes. The spheroidal pink shaded regions represent the anisotropy of $g$-factor. $(\mathbf{e}, \mathbf{f})$ Angular variation of magnetic torque (left axis) at 1.8 and $0.3 \mathrm{~K}$ (red and blue filled circles, respectively) and the $g$-factor determined by the EPR at $4.2 \mathrm{~K}$ (open circles, right axis) when the magnetic field is rotated within the $c^{\star}-a$ plane (e) and $c^{\star}-b$ plane (f). The solid lines are fits to the $\sin 2 \theta$ dependence.

out (see Methods). Our torque magnetometry down to low temperatures $(30 \mathrm{mK})$ up to high fields $(32 \mathrm{~T})$ reveals distinct residual paramagnetic susceptibility comparable to that in a half-filled 2D metal, showing 'magnetic' gapless excitations in the QSL of $\mathrm{EtMe}_{3} \mathrm{Sb}\left[\mathrm{Pd}(\mathrm{dmit})_{2}\right]_{2}$. We further demonstrate that changing the degree of frustration by a deuteration has a small effect on the susceptibility, indicating the emergence of an extended quantum critical phase in which the low-lying excitations are fermions with a Fermi surface.

\section{Results}

Electron paramagnetic resonance measurements. To determine the $g$-factor anisotropy, which is a fundamental property to discuss the magnetic anisotropy, we first measure electron paramagnetic resonance (EPR) as a function of field angle. The principal axes of the gyromagnetic ratio ( $g$-factor) of $\mathrm{EtMe}_{3} \mathrm{Sb}\left[\mathrm{Pd}(\mathrm{dmit})_{2}\right]_{2}, p, q$ and $r$ (see Fig. 1c,d), are deduced from the EPR measurements at $4.2 \mathrm{~K}$. 

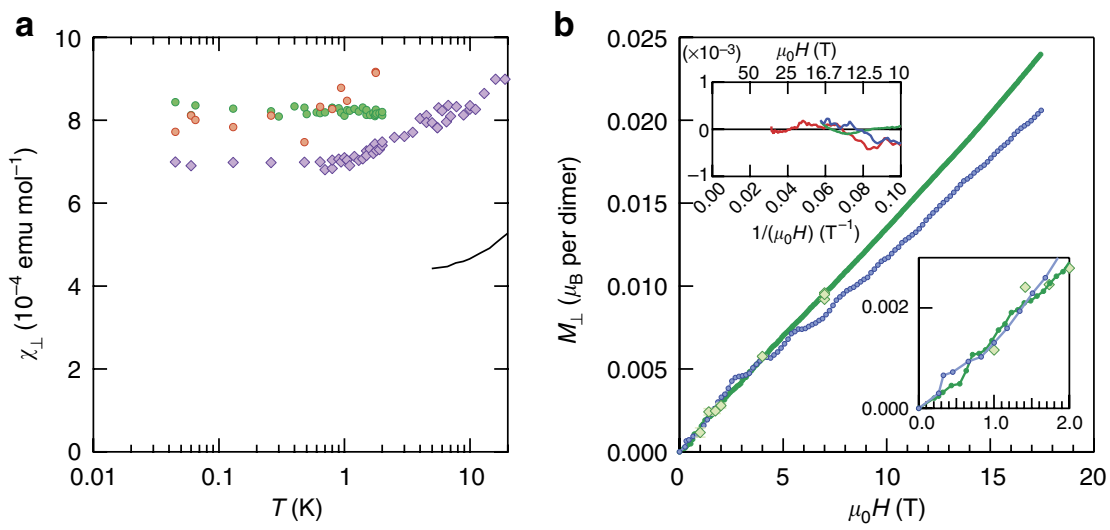

Figure 2 | Magnetic susceptibility and magnetization in the QSL state of EtMe $\mathbf{S}_{\mathbf{3}} \mathbf{S b}\left[\mathrm{Pd}(\mathrm{dmit})_{2}\right]_{\mathbf{2}}$ and its deuterated compound. (a) Temperature dependence of $\chi_{\perp}$ of two crystals of pristine material ( $h_{9}$-dmit (A) green circles, $h_{9}$-dmit (B) orange circles) and a deuterated sample ( $d_{9}$ - $d m i t$ purple diamonds) determined by the torque curves at 7T. The black solid line is the susceptibility data of polycrystalline sample measured by the SQUID magnetometer ${ }^{26}$, where the large Curie contribution from impurities has been subtracted. (b) Field dependence of magnetization $M_{\perp}$ determined from $\chi_{\perp}$ of pristine ( $h_{9}$-dmit $(A)$ green circles) and deuterated ( $d_{9}$-dmit blue circles) samples. The data shown as filled circles $(T=30 \mathrm{mK})$ were taken by sweeping field at a fixed angle $\theta$. The light green diamonds show the amplitude of the whole torque curves $\tau(\theta)$ at $50 \mathrm{mK}$ for the $h_{9}$-dmit $(A)$ sample. The lower inset shows $M_{\perp}$ in the low field region. The upper inset is the deviation from the high-filed linear dependence plotted against the inverse field. The data up to $32 \mathrm{~T}$ (for $\mathrm{h}_{9}$-dmit (C) red line) show no apparent quantum oscillations.

The angular dependence of the $g$-factor is shown in Fig. 1e,f (open circles). As the principle axes of the $g$-tensor virtually coincide with that of the molecule, the anisotropy of the g-factor is likely given by the spin-orbit coupling as found in other organic materials ${ }^{34,35}$ (see Methods).

Magnetic torque measurements. Figure le,f depicts the magnetic torque curves of $\mathrm{EtMe}_{3} \mathrm{Sb}\left[\mathrm{Pd}(\mathrm{dmit})_{2}\right]_{2}\left(\mathrm{~h}_{9}\right.$-dmit) when the magnetic field of $\mu_{0} H=7 \mathrm{~T}$ is rotated within the $a c^{\star}$ and $b c^{\star}$ plane, where $\theta$ is the polar angle measured from the $c^{\star}$ axis. We note that although the principle axes of the $g$-tensor do not coincide with the crystallographic axes (Fig. 1c,d), the torque crosses zero exactly at the minimum and maximum positions of the $g$-factor for both field rotation at all temperatures, indicating that the torque signal reflects the anisotropy of the $g$-factor. Assuming that the $g$-factor is temperature independent at low temperatures, we determined $\chi_{\perp}$ and $M_{\perp}$ (see Methods).

Figure 2a,b depicts the temperature dependence of $\chi_{\perp}$ at $\mu_{0} H=7 \mathrm{~T}$ and the field dependence of $M_{\perp}$ at $T=30 \mathrm{mK}$, respectively, for pristine crystal ( $\mathrm{h}_{9}$-dmit) and its deuterated compound $\left(\mathrm{d}_{9}\right.$-dmit), which has different degrees of geometrical frustration ${ }^{29}$. The absolute value of $\chi_{\perp}$ is nearly 1.5 times larger than $\chi$ of polycrystalline samples of $h_{9}$-dmit determined by the SQUID magnetometer (Fig. 2a).

We also measured the magnetic torque of the pristine sample $\left(\mathrm{h}_{9^{-}}\right.$ dmit (C)) in magnetic fields up to $32 \mathrm{~T}$ to search a quantum oscillation. After subtracting the background magnetization signal determined by a linear approximation to the data $>12 \mathrm{~T}$, we plot the data as a function of $1 / H$ as the red line in the upper inset of Fig. 2b. No discernible oscillation is observed within the experimental resolution.

\section{Discussion}

The most notable features are (i) $\chi_{\perp}$ is temperature independent $<2 \mathrm{~K}$ and remains finite in zero temperature limit (Fig. 2a) and (ii) $M_{\perp}$ increases nearly linearly with the applied field up to $17 \mathrm{~T}$ (Fig. 2b). In the dmit materials the orbital paramagnetic (Van Vleck) susceptibility is very small (see Methods), and thus the spin (Pauli) paramagnetism should be responsible for the observed temperature-independent and field-linear paramagnetic response. These results in the low-temperature limit provide direct evidence of the presence of low-lying gapless 'magnetic' excitations. It is also clear that the gapless excitations observed in the thermal conductivity ${ }^{27}$ and the heat capacity ${ }^{29}$ measurements contain the magnetic ones. As the spin gap $\Delta$ is inversely proportional to the magnetic correlation length $\xi$, this spin-gapless QSL state has infinite $\xi$. Therefore, the presence of the gapless magnetic excitations is of crucially importance, as it immediately indicates that the QSL is in a critical state where the spin-spin correlation function decays with distance $r$ in an anomalous non-exponential manner: a simple example is the power-law decay $\left\langle S^{z}(r) S^{z}(0)\right\rangle \propto r^{-\eta}$ with $\eta>0$ for the so-called algebraic spin liquid ${ }^{5}$.

There are two possible phase diagrams for the QSL as a function of frustration, as illustrated in Fig. 3a,b. With increasing frustration, a magnetically ordered phase with broken symmetry is destroyed at a critical value, beyond which a QSL phase without broken symmetry appears. The first case is that a gapless QSL with infinite $\xi$ emerges only at a quantum critical point ${ }^{36}$, beyond which a spin-gapped QSL with finite $\xi$ appears (Fig. 3a). In the gapped QSL, its spin correlation function decays exponentially with distance as $\exp (-r / \xi)$ except at the quantum critical point. The second case is that there is an extended critical 'phase', where the gapless QSL with infinite correlation length is stably present (Fig. 3b). These two cases can be distinguished by comparing $\chi_{\perp}$ of $\mathrm{h}_{9}$-dmit and $\mathrm{d}_{9}$-dmit at $k_{\mathrm{B}} T \ll J$, because deuteration of the Me groups in $\mathrm{EtMe}_{3} \mathrm{Sb}\left[\mathrm{Pd}(\mathrm{dmit})_{2}\right]_{2}$ changes the degrees of geometrical frustration by reducing $t^{\prime} / t$ (ref. 29). As shown in Fig. 2a,b, both $h_{9^{-}}$and $d_{9}$-dmit crystals exhibit essentially the same paramagnetic behaviours with no spin gap, indicating that both materials are in a critical state down to $k_{\mathrm{B}} T \sim J / 10,000$. These results lead us to conclude the presence of an extended quantum critical phase of the spin-gapless QSL (Fig. 3b).

The presence of a stable QSL 'phase' with gapless magnetic excitations is hard to explain in the conventional bosonic picture. Within this picture, when the magnetic long-range order is established, the gapless Nambu-Goldstone bosons, that is, magnons, would arise as the consequence of the spontaneous symmetry breaking of the spin $\mathrm{SU}(2)$ symmetry. When the system evolves into the QSL state with no symmetry breaking, the common wisdom is that both of the magnetic order and Nambu-Goldstone bosons disappear at the same time. This implies that the presence of gapless QSL phase has some exotic elementary excitations. Among them, 'spinon' 

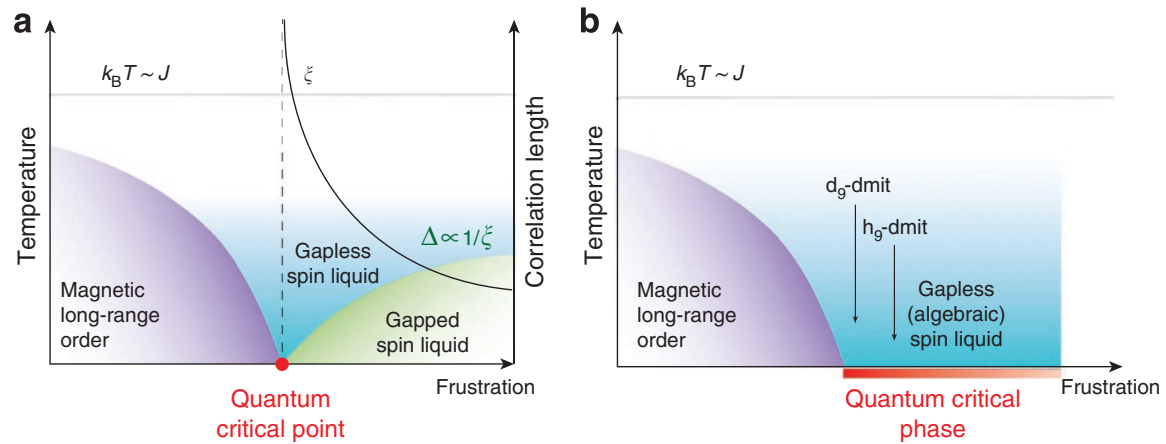

Figure $\mathbf{3}$ | Schematic phase diagrams of temperature versus frustration for Mott insulating antiferromagnets with 2D triangular lattice. The QSL appears in two distinctly different ways. (a) The ordered ground state (with no gap) immediately evolves into a gapped QSL. A gapless QSL, in which the spin correlation decays algebraically with distance, appears only at the quantum critical point (QCP), which is the border between the ordered state and the gapped QSL state. In this case, the observation of the critical gapless QSL requires a fine tuning of the material parameters. (b), An extended quantum critical 'phase', where the gapless QSL with infinite correlation length appears beyond a critical value. The fact that both $h_{9}$-dmit and $d_{9}$-dmit with different degrees of frustration exhibit essentially the same paramagnetic behaviours with no spin gap indicates that both materials are in the same critical state. This leads us to conclude the presence of a quantum critical phase (b), rather than a QCP (a), in $\mathrm{EtMe}_{3} \mathrm{Sb}\left[\mathrm{Pd}(\mathrm{dmit})_{2}\right]_{2}$.

excitation, which is a chargeless spin-1/2 quasiparticle (half of the magnons), has been discussed extensively. In theories of the QSL with the bosonic spinons, the magnetic order is described by the Bose-Einstein condensation ${ }^{37}$ of the bosonic spinons. When the magnetic order is destroyed or the Bose-Einstein condensation disappears, the bosonic spinons in the resulting QSL can only be gapless at the critical point beyond which they become gapped ${ }^{6}$. The presence of an extended region of spin-gapless QSL phase at zero or very low temperatures, therefore, would suggest that the underlying QSL phase may be better described by the QSL with fermionic spinons ${ }^{7,9}$.

Indeed, the presence of the gapless magnetic excitations bears some resemblance to the elementary excitations in the spin channel of metals with Fermi surface, that is, Pauli paramagnetism. Here, we examine a thermodynamic test simply by analysing the data in accordance with the assumptions that the elementary quasiparticles in the QSL phase possess Fermi surface, even though the system is an insulator. The Pauli susceptibility is given by $\chi_{\perp}=(1 / 4) g_{*}^{2} \mu_{\mathrm{B}}^{2} D\left(\varepsilon_{\mathrm{F}}\right)$, where $\varepsilon_{\mathrm{F}}$ is the Fermi energy, $D\left(\varepsilon_{\mathrm{F}}\right)$ is the density of states and $\mu_{\mathrm{B}}$ is the Bohr magneton. In $2 \mathrm{D}$ systems, $D\left(\varepsilon_{\mathrm{F}}\right)=n / \varepsilon_{\mathrm{F}}$, where $n$ is the number of fermion (spin) per volume. Using $\chi_{\perp}=8.0(5) \times$ $10^{-4} \mathrm{emu} \mathrm{mol}^{-1}$ (Fig. 2a), the specific heat coefficient of the fermionic excitations, which corresponds to the Sommerfeld constant in metals, yielded by the equation $\gamma=(1 / 3) \pi^{2} k_{\mathrm{B}}^{2} D\left(\varepsilon_{\mathrm{F}}\right)$, is estimated to be $\gamma \approx 56 \mathrm{~mJ} \mathrm{~K}^{-2} \mathrm{~mol}^{-1}$. This value is of the same order of magnitude as the values reported by the heat capacity measurements ${ }^{29}$. Provided that a substantial portion of the gapless excitations observed by specific heat is magnetic, the $\gamma$ and $\chi_{\perp}$ values result in a Wilson ratio $R_{\mathrm{W}}$ close to unity $\left(R_{\mathrm{W}}=2.83\right.$ (1.41) for the pristine (deuterated) sample), which is a basic properties of metals. Moreover, it has been predicted ${ }^{38}$ that the Fermi temperature of the spinon is roughly of the order of magnitude of $J / k_{\mathrm{B}}(\sim 250 \mathrm{~K})$. The Fermi temperature, given by the relation $T_{\mathrm{F}}=\varepsilon_{\mathrm{F}} / k_{\mathrm{B}}=g_{*}^{2} \mu_{\mathrm{B}}^{2} / 4 \chi_{\perp} k_{\mathrm{B}}$, is estimated to be $480 \pm 30 \mathrm{~K}$, which is in the same order as $J / k_{\mathrm{B}}$. Thus, although the present simple estimations should be scrutinized, all thermodynamic quantities appear to be quantitatively consistent with the theory of the QSL that possesses a spinon Fermi surface. In $\mathrm{d}_{9}$-dmit, while $\gamma$-value is enhanced by nearly twice, $\chi_{\perp}$ is reduced by nearly $15 \%$, compared with $\mathrm{h}_{9}$-dmit. This may indicate that nonmagnetic spin-singlet excitations, which enter not in $\chi_{\perp}$ but in $\gamma$, are enhanced very close to the magnetic end point. This might be a novel phenomenon unanticipated by present theories, which will deserve further studies.
There are several types of theories that predict the gapless QSL with spinon Fermi surface. Some of these theories, which include Amperian pairing ${ }^{13}, d$-wave pairing ${ }^{14}$ and gapless triplet pairing ${ }^{15}$, predict an instability of the spinon Fermi surface at low temperatures, which accompany a phase transition. Here, we comment on these theories. Although the NMR measurements report an anomaly at $1 \mathrm{~K}$ (ref. 28) and discuss possible instabilities at low temperatures ${ }^{13-15}$, no anomaly is observed in the magnetic susceptibility, which is consistent with the thermal conductivity ${ }^{27}$ and specific heat measurements ${ }^{29}$. This indicates that the NMR anomaly at $1 \mathrm{~K}$ is not a signature of the thermodynamic transition, but a phenomenon that may be related to the slow spin dynamics of the QSL. Nevertheless, more stringent test of phase transition should be examined using experimental probes sensitive to rotational symmetry breaking 32,33 , which deserves further studies. We also note that the recent theory of Majorana fermions with a Fermi surface $^{16}$ predicts the Wilson ratio that is close to our observation. However, the suppression of the susceptibility in high magnetic fields predicted by this theory is not observed in this experiment at least up to $17 \mathrm{~T}$.

The most direct evidence of the presence of the Fermi surface may be given by the quantum oscillation measurements ${ }^{39}$. However, as shown in the inset in Fig. 2b, we find an extended paramagnetic response up to the highest field, but no discernible oscillation is observed within the experimental resolution (upper inset in Fig. 2b). We find that our estimation of the mobility of the gapless excitation strongly suggests that the impurity scattering effect is not the main origin of the absence of the quantum oscillations (see Methods). A possible explanation for this is that the gauge flux, which causes the quantum oscillation for the spinons, only weakly couples to the applied magnetic field or becomes unstable under a small magnetic field ${ }^{39}$. This seems to be consistent with the fact that our torque curves can be described only by the $g$-factor anisotropy, without showing any contribution from the orbital (spinon) motion in the $2 \mathrm{D}$ plane. We also point out that the thermal Hall effect in this material ${ }^{27}$ is negligibly small compared with a theoretical suggestion ${ }^{38}$, which may be originated from the same physics.

The presently revealed extended Pauli-paramagnetic quantum phase bears striking resemblance to metals with Fermi surface rather than insulators even though the charge degrees of freedom are frozen. We also emphasise that the presence of such a novel quantum phase might provide profound implications on the physics of other class of strongly interacting many-body systems in the 
vicinity of the end point of long-range order as well as the exotic quantum spin systems.

\section{Methods}

Sample preparation. Single crystals of $\mathrm{EtMe}_{3} \mathrm{Sb}\left[\mathrm{Pd}(\mathrm{dmit})_{2}\right]_{2}\left(\mathrm{Et}=\mathrm{C}_{2} \mathrm{H}_{5}\right.$, $\mathrm{Me}=\mathrm{CH}_{3}$, dmit = 1,3-dithiole-2-thione-4,5-dithiolate) were grown by the airoxidation method. This material has a layered structure (space group $C 2 / c$ ) with the lattice parameters $a=14.515 \AA, b=6.408 \AA$, and the inter-layer distance $d=c^{\star} / 2=18.495 \AA$. The deuterated crystals of $\mathrm{d}_{9}-\mathrm{EtMe}_{3} \mathrm{Sb}\left[\mathrm{Pd}(\mathrm{dmit})_{2}\right]_{2}$ were prepared by the same method using $\left(\mathrm{Et}\left(\mathrm{CD}_{3}\right)_{3} \mathrm{Sb}\right)_{2}\left[\mathrm{Pd}(\mathrm{dmit})_{2}\right]$. The deuterium atoms were introduced by CD3I $(99.5 \% \mathrm{D})$. Typical sample size is $\sim 1 \times 1 \times 0.05 \mathrm{~mm}$.

Torque measurements. All magnetic torque measurements were carried out by attaching one single crystal to a cantilever with a tiny amount of grease. Piezo-resistive cantilevers were used in the measurements at the Kyoto University $\left(\mu_{0} H<7 \mathrm{~T}, T>0.3 \mathrm{~K}\right)$ and at Tsukuba Magnet Laboratories, NIMS $\left(\mu_{0} H<17 \mathrm{~T}\right.$, $30 \mathrm{mK} \leq T \leq 1 \mathrm{~K})$. In the measurement at high magnetic field facility at Grenoble $(30 \mathrm{mK} \leq T \leq 1 \mathrm{~K})$, magnetic fields up to $32 \mathrm{~T}$ were provided by a resistive magnet and a capacitance-sensing metallic cantilever was used. To determine the absolute value of $\chi_{\perp}$ (Fig. 2a), sensitivities of the each cantilever were calibrated in situ by detecting $\sin \theta$ oscillation owing to the sample mass at zero field. Thermal equilibrium between samples and cryostat at the lowest temperature was ensured by immersing the whole setup into the mixture of the dilution refrigerator.

Electron paramagnetic resonance measurements. All EPR measurements have been performed in RIKEN by using a conventional X-band EPR spectrometer (JEOL, JES-RE3X) equipped with a continuous He-flow cryostat. All EPR spectra have a symmetric Lorentzian line shape with the line width of $3 \mathrm{mT}$. The angular dependence measurements of the $g$-value was carried out using the same single crystal at $4.2 \mathrm{~K}$, and the $g$-value for each angle has been precisely obtained from the correction using the $\mathrm{Mn}^{2+}$ field marker. It should be noted that detail mechanism of the anisotropy of the $g$-tensor has been discussed in other Pd(dmit $)_{2}$ compounds $^{34}$, (TMTTF) ${ }_{2} X$ (ref. 35), and inorganic spin chain materials ${ }^{40}$ from the dependence of the line width on the sample angle, the frequency and the temperature. Such comprehensive EPR studies of $\mathrm{EtMe}_{3} \mathrm{Sb}\left[\mathrm{Pd}(\mathrm{dmit})_{2}\right]_{2}$ remain as a future work.

Determination of susceptibility from the torque measurements. Here, we describe how we determine the uniform susceptibility $\chi_{\perp}$ and the magnetization $M_{\perp}$ from our torque measurements.

By adopting the principal axes of the $g$-factor $(p, q$ and $r=p \times q$ as shown in Fig. 1c,d) as orthogonal coordinate system, the spin susceptibility tensor can be diagonalized as

$$
M=\left(\begin{array}{ccc}
\chi_{p p} & 0 & 0 \\
0 & \chi_{q q} & 0 \\
0 & 0 & \chi_{r r}
\end{array}\right) H
$$

The torque detected by the cantilever method is a component perpendicular to the field-rotating plane (Fig. $1 \mathrm{~b}$ ). For the $c^{\star}-a$ rotation this is given as

$$
\begin{aligned}
\tau_{c^{*}-a}(\theta) & =\left(c^{*} \times a\right) \cdot(M \times H) \\
& =\frac{1}{2} \mu_{0} H^{2} V\left(\chi_{q q}-\chi_{p p}\right) \sin 2\left(\theta+\theta_{0}\right),
\end{aligned}
$$

and for the $c^{*}-b$ rotation we obtain

$$
\begin{aligned}
\tau_{c^{*}-b}(\theta) & =\left(c^{*} \times b\right) \cdot(M \times H) \\
& =\frac{1}{2} \mu_{0} H^{2} V\left(\chi_{p p} \sin ^{2} \theta_{0}+\chi_{q q} \cos ^{2} \theta_{0}-\chi_{r r}\right) \sin 2 \theta .
\end{aligned}
$$

These equations show that the torque should have a sinusoidal shape with twofold oscillation crossing zero at the maximum and minimum positions of the $g$-factor. This is exactly what we observed (Fig. 1e,f). This indicates that the susceptibility can be diagonalized as in equation (1) and that the anisotropy of the $g$-factor is the origin of the anisotropy of the susceptibility in this spin liquid system.

Having established that the magnetic susceptibility reflects the anisotropy of the $g$-factor, we assume that the susceptibility is proportional to the square of the $g$-factor $\left(\chi_{i i}=g_{i i}^{2} \tilde{\chi}\right)$ as in conventional paramagnetic systems. Then $\chi_{i j}$ can be obtained by the reduced susceptibility $\tilde{\chi}$, which is determined from $\tau_{c^{*}-a}$ and $\tau_{c^{*}-b}$ as

$$
\begin{aligned}
& \tau_{c^{*}-a}(\theta)=\frac{1}{2} \mu_{0} H^{2} V\left(g_{q q}^{2}-g_{p p}^{2}\right) \tilde{\chi} \sin 2\left(\theta+\theta_{0}\right) \\
& \tau_{c^{*}-b}(\theta)=\frac{1}{2} \mu_{0} H^{2} V\left(g_{p p}^{2} \sin ^{2} \theta_{0}+g_{q q}^{2} \cos ^{2} \theta_{0}-g_{r r}^{2}\right) \tilde{\chi} \sin 2 \theta,
\end{aligned}
$$

where the principal values of the $g$-factor and $\theta_{0}$ are provided by the EPR measurements (Fig. 1e,f) as $g_{p p}=1.9963, g_{q q}=2.0325, g_{r r}=2.0775, g_{c^{*} c^{*}}=2.0217$ and $\theta_{0}=32$ degree. The susceptibility for the magnetic field perpendicular to the basal plane is, therefore, given by

$$
\chi_{\perp}=g_{c^{*} c^{*}}^{2} \tilde{\chi}, \quad M_{\perp}=\chi_{\perp} H .
$$

The two independent measurements $\tau_{*^{*}}$ and $\tau_{\mathcal{*}_{-} b}$ give quantitatively consistent results for $\chi_{\perp}$ (red and green circles in ${ }^{c}$ Fig. $-a$ a, respectively), confirming the validity of our analysis.

Origin of the paramagnetic susceptibility. The obtained $\chi_{\perp}(\mathrm{T})$ in the spin-liquid state of EtMe $3 \mathrm{Sb}\left[\mathrm{Pd}(\mathrm{dmit})_{2}\right]_{2}$ is nearly temperature-independent at low temperatures below $\sim 2 \mathrm{~K}$. This immediately indicates that in the torque measurements, the Curie contribution from the free spins of the impurities is almost exactly cancelled out. This indicates that such free spins are not subject to the $g$-factor anisotropy, which may be explained if the impurity spins reside at the $\mathrm{EtMe}_{3} \mathrm{Sb}$ cation site between the dmit layers. The absence of the Curie term is also confirmed from the field-linear magnetization without saturation in a wide field range covering large $B / T$ ratios, which cannot be fitted by the Brillouin function.

The paramagnetic temperature-independent susceptibility, in general, may be originated from the orbital Van Vleck susceptibility and the spin Pauli susceptibility. However, the Van Vleck term is found to be negligibly small in the closely related isostructural material $\mathrm{Et}_{2} \mathrm{Me}_{2} \mathrm{Sb}\left[\mathrm{Pd}(\mathrm{dmit})_{2}\right]_{2}$ with slightly different cations $\mathrm{Et}_{2} \mathrm{Me}_{2} \mathrm{Sb}$. In this material, a large spin gap is formed below the first-order transition at $\sim 70 \mathrm{~K}$, which has been attributed to the charge disproportionation in $\left[\mathrm{Pd}(\mathrm{dmit})_{2}\right]_{2}$ anions. The susceptibility jumps from the value close to the present $\mathrm{EtMe}_{3} \mathrm{Sb}\left[\mathrm{Pd}(\mathrm{dmit})_{2}\right]_{2}$ case at high temperature to almost zero below the transition, indicating the absence of the Van Vleck paramagnetic susceptibility ${ }^{41}$. This strongly suggests that the sizable paramagnetic temperature-independent susceptibility in the spin liquid state of $\mathrm{EtMe}_{3} \mathrm{Sb}\left[\mathrm{Pd}(\mathrm{dmit})_{2}\right]_{2}$ can be fully attributed to the spin Pauli susceptibility. We also note that the diamagnetic Landau susceptibility, if present, should give a very minor correction to the Pauli susceptibility, because we did not observe the quantum oscillations at high magnetic fields.

Estimation of the mobility of the gapless excitation. An important requirement to observe the quantum oscillation in the torque (de Haas-van Alphen effect) in a metal is a long mean-free path $\ell=v_{\mathrm{F}} \tau\left(\nu_{\mathrm{F}}\right.$ is the Fermi velocity and $\tau$ is the scattering time) to satisfy that $\omega_{c} \tau=e B \tau / m^{*}=\mu B$ exceeds unity, where $\omega_{c}$ is the cyclotron frequency, $m^{*}$ is the effective mass and $\mu$ is the mobility. In a simple metal, the mobility can be estimated by using the residual thermal conductivity $\kappa_{0} / T$, the Sommerfeld constant $\gamma$, and the Fermi energy $\varepsilon_{\mathrm{F}}$ through

$$
\mu=\frac{e \tau}{m^{*}}=\frac{3 \kappa_{0} / T}{2 \gamma \varepsilon_{\mathrm{F}}}
$$

By using $\kappa_{0} / T \approx 0.2 \mathrm{~W} \mathrm{~K}^{-2} \mathrm{~m}^{-1}$ (ref. 27), $\gamma \approx 20 \mathrm{~mJ} \mathrm{~K}^{-2} \mathrm{~mol}^{-1}$ (ref. 29) and $\varepsilon_{\mathrm{F}} / k_{\mathrm{B}} \sim 480 \mathrm{~K}$ estimated by the present susceptibility measurements, we can roughly estimate $\mu \sim 0.18 \mathrm{~T}^{-1}$ in the spin-liquid state of $\mathrm{EtMe}_{3} \mathrm{Sb}\left[\mathrm{Pd}(\mathrm{dmit})_{2}\right]_{2}$. This yields $\omega_{\mathrm{c}} \tau>5$ at $32 \mathrm{~T}$, which strongly suggests that the impurity scattering effect is not the main origin of the absence of the quantum oscillations.

\section{References}

1. Balents, L. Spin liquids in frustrated magnets. Nature 464, 199-208 (2010)

2. Anderson, P. W. Resonating valence bonds: a new kind of insulator. Mater. Res. Bull. 8, 153-160 (1973).

3. Lee, P. A., Nagaosa, N. \& Wen, X. G. Doping a Mott insulator: Physics of hightemperature superconductivity. Rev. Mod. Phys. 78, 17-85 (2006).

4. Moessner, R. \& Sondhi, S. L. Resonating valence bond phase in the triangular lattice quantum dimer model. Phys. Rev. Lett. 86, 1881-1884 (2001).

5. Wen, X.- G. Quantum orders and symmetric spin liquids. Phys. Rev. B 65, 165113 (2002)

6. Qi, Y., Xu, C. \& Sachdev, S. Dynamics and transport of the $Z_{2}$ spin liquid: Application to $\kappa$-(ET) ${ }_{2} \mathrm{Cu}_{2}(\mathrm{CN})_{3}$. Phys. Rev. Lett. 102, 176401 (2009).

7. Block, M. S., Sheng, D. N., Motrunich, O. I. \& Fisher, M. P. A. Spin bose-metal and valence bond solid phases in a spin-1/2 model with ring exchanges on a four-leg triangular ladder. Phys. Rev. Lett. 106, 157202 (2011). 
8. Motrunich, O. I. Variational study of triangular lattice spin-1/2 model with ring exchanges and spin liquid state in $\kappa-(\mathrm{ET})_{2} \mathrm{Cu}_{2}(\mathrm{CN})_{3}$. Phys. Rev. B 72, 045105 (2005).

9. Lee, S.- S. \& Lee, P. A. U(1) gauge theory of the Hubbard model: spin liquid states and possible application to $\kappa-(\mathrm{ET})_{2} \mathrm{Cu}_{2}(\mathrm{CN})_{3}$. Phys. Rev. Lett. 95, 036403 (2005).

10. Potter, A. C., Barkeshli, M., McGreevy, J. \& Senthil, T. Quantum spin liquids and the metal-insulator transition in doped semiconductors. Preprint at http://arXiv:1204.1342 (2012).

11. Misguich, G., Lhuillier, C., Bernu, B. \& Waldtmann, C. Spin-liquid phase of the multiple-spin exchange Hamiltonian on the triangular lattice. Phys Rev. B. 60, 1064-1074 (1999).

12. Lawler, M. J., Paramekanti, A., Kim, Y. B. \& Balents, L. Gapless spin liquids on the three dimensional hyper-kagome lattice of $\mathrm{Na}_{4} \mathrm{Ir}_{3} \mathrm{O}_{8}$. Phys. Rev. Lett. 101, 197202 (2008).

13. Lee, S.- S., Lee, P. A. \& Senthil, T. Amperean pairing instability in the U(1) spin liquid state with fermi surface and application to $\kappa-(\mathrm{ET})_{2} \mathrm{Cu}_{2}(\mathrm{CN})_{3}$. Phys. Rev. Lett. 98, 067006 (2007).

14. Grover, T., Trivedi, N., Senthil, T. \& Lee, P. A. Weak Mott insulators on the triangular lattice: possibility of a gapless nematic quantum spin liquid. Phys. Rev. B. 81, 245121 (2010).

15. Galitski, V. \& Kim, Y. B. Spin-triplet pairing instability of the spinon Fermi surface in a $U(1)$ spin liquid. Phys. Rev. Lett. 99, 266403 (2007).

16. Biswas, R. R., Fu, L., Laumann, C. R. \& Sachdev, S. SU(2)-invariant spin liquids on the triangular lattice with spinful Majorana excitations. Phys. Rev. B. 83, 245131 (2011)

17. Morita, H., Watanabe, S. \& Imada, M. Nonmagnetic insulating states near the Mott transitions on lattices with geometrical frustration and implications for $\kappa$-(ET) ${ }_{2} \mathrm{Cu}_{2}(\mathrm{CN})_{3}$. J. Phys. Soc. Jpn. 71, 2109-2112 (2002).

18. Kyung, B. \& Tremnlay, A.- M. S. Mott transition, antiferromagnetism, and $d$-wave superconductivity in two-dimensional organic conductors. Phys. Rev. Lett. 97, 046402 (2006).

19. Yoshioka, T., Koga, A. \& Kawakami, N. Quantum phase transitions in the Hubbard model on a triangular lattice. Phys. Rev. Lett. 103, 036401 (2009).

20. Shimizu, Y., Miyagawa, K., Kanoda, K., Maesato, M. \& Saito, G. Emergence of inhomogeneous moments from spin liquid in the triangular-lattice Mott insulator $\kappa-(\mathrm{ET})_{2} \mathrm{Cu}_{2}(\mathrm{CN})_{3}$. Phys. Rev. B 73, 140407 (2006).

21. Yamashita, S. et al. Thermodynamic properties of a spin- $1 / 2$ spin-liquid state in a $\kappa$-type organic salt. Nat. Phys. 4, 459-462 (2008).

22. Yamashita, M. et al. Thermal-transport measurements in a quantum spin-liquid state of the frustrated triangular magnet $\kappa-(\mathrm{ET})_{2} \mathrm{Cu}_{2}(\mathrm{CN})_{3}$. Nat. Phys. 5, 44-47 (2009).

23. Manna, R. S., de Souza, M., Brühl, A., Schlueter, J. A. \& Lang, M. Lattice effects and entropy release at the low-temperature phase transition in the spin-liquid candidate $\kappa$ - $(\mathrm{ET})_{2} \mathrm{Cu}_{2}(\mathrm{CN})_{3}$. Phys. Rev. Lett. 104, 016403 (2010).

24. Nakajima, S. et al. Microscopic phase separation in triangular-lattice quantum spin magnet $\kappa$-(BEDT-TTF $)_{2} \mathrm{Cu}_{2}(\mathrm{CN})_{3}$ probed by muon spin relaxation. J. Phys. Soc. Jpn. 81, 063706 (2012).

25. Pratt, F. L. et al. Magnetic and non-magnetic phases of a quantum spin liquid. Nature 471, 612-616 (2011).

26. Itou, T., Oyamada, A., Maegawa, S., Tamura, M. \& Kato, R. Quantum spin liquid in the spin-1/2 triangular antiferromagnet $\mathrm{EtMe}_{3} \mathrm{Sb}\left[\mathrm{Pd}(\mathrm{dmit})_{2}\right]_{2}$. Phys. Rev. B 77, 104413 (2008).

27. Yamashita, M. et al. Highly mobile gapless excitations in a two-dimensional candidate quantum spin liquid. Science 328, 1246-1248 (2010).
28. Itou, T., Oyamada, A., Maegawa, S. \& Kato, R. Instability of a quantum spin liquid in an organic triangular-lattice antiferromagnet. Nat. Phys. 6, 673-676 (2010).

29. Yamashita, S. et al. Gapless spin liquid of an organic triangular compound evidenced by thermodynamic measurements. Nat. Commun. 2, 275 (2011)

30. Kanoda, K. \& Kato, R. Mott physics in organic conductors with triangular lattices. Annu. Rev. Condens. Matter Phys. 2, 167-188 (2011).

31. Huse, D. A. \& Elser, V. Simple variational wave functions for two-dimensional Heisenberg spin-1/2 antiferromagnets. Phys. Rev. Lett. 60, 2531-2534 (1988).

32. Okazaki, R. et al. Rotational symmetry breaking in the hidden-order phase of $\mathrm{URu}_{2} \mathrm{Si}_{2}$. Science 331, 439-442 (2011)

33. Kasahara, S. et al. Electronic nematicity above the structural and superconducting transition in $\mathrm{BaFe}_{2}\left(\mathrm{As}_{1-x} \mathrm{P}_{x}\right)_{2}$. Nature 486, 382-385 (2012).

34. Nakamura, T., Takahashi, T., Aonuma, S. \& Kato, R. $g$-Tensor analyses of $\beta^{\prime}$-type Pd(dmit) ${ }_{2}$ metal complexes. Mol. Cryst. Liq. Cryst. 379, 53-58 (2002).

35. Yasin, S. et al. Broken magnetic symmetry due to charge-order ferroelectricity discovered in (TMTTF) ${ }_{2} X$ salts by multifrequency ESR. Phys. Rev. B 85, 144428 (2012).

36. Sachdev, S. Quantum Phase Transitions (Cambridge Univ. Press, Cambridge, 1999).

37. Giamarchi, T., Rüegg, C. \& Tchernyshyov, O. Bose-Einstein condensation in magnetic insulators. Nat. Phys. 4, 198-204 (2008)

38. Katsura, H., Nagaosa, N. \& Lee, P. A. Theory of the thermal Hall effect in quantum magnets. Phys. Rev. Lett. 104, 066403 (2010)

39. Motrunich, O. I. Orbital magnetic field effects in spin liquid with spinon Ferm sea: possible application to $\kappa$ - $(\mathrm{ET})_{2} \mathrm{Cu}_{2}(\mathrm{CN})_{3}$. Phys. Rev. B 73, 155115 (2006).

40. Herak, M. et al. Symmetric and antisymmetric exchange anisotropies in quasione-dimensional $\mathrm{CuSe}_{2} \mathrm{O}_{5}$ as revealed by ESR. Phys. Rev. B 84, 184436 (2011).

41. Tamura, M. \& Kato, R. Spin-1/2 Heisenberg antiferromagnets on anisotropic triangular lattice, $\left[\mathrm{Pd}(\mathrm{dmit})_{2}\right]$ salts: How do they release frustration? Polyhedron 24, 2817-2820 (2005)

\section{Acknowledgements}

We thank fruitful discussion with L. Balents, S. Fujimoto, T. Goto, M. Imada, N. Kawakami, Y.B. Kim, P.A. Lee, R. Moessner, N. Nagaosa, T. Sasaki, T. Senthil and K. Totsuka. This research has been supported through Grant-in-Aid for the Global COE program 'The Next Generation of Physics, Spun from Universality and Emergence' from the Ministry of Education, Culture, Sports, Science and Technology (MEXT) of Japan, KAKENHI from JSPS and grant-in-aid for Scientific Research on Innovative Areas (nos. 20110003 and 23110716) from the MEXT. Part of this work has been supported by EuroMagNET II under the EU contract.

\section{Author contributions}

D.W., M.Y., S.T., I.S., T.T. and S.U. made torque measurements. Y.O., H.M.Y. and R.K. grew and characterized the crystals. D.W. and M.Y. analysed the data. T.S. and Y.M. conceived and designed the project. M.Y., K.B., T.S. and Y.M. wrote the manuscript with input from all the authors. D.W. and M.Y. contributed equally to this work.

\section{Additional Information}

Competing financial interests: The authors declare no competing financial interests.

Reprints and permission information is available online at http://npg.nature.com/ reprintsandpermissions

How to cite this article: Watanabe, D. et al. Novel Pauli-paramagnetic quantum phase in a Mott insulator. Nat. Commun. 3:1090 doi: 10.1038/ncomms2082 (2012). 